\title{
A simple model for the normal stress distribution of the Great Pyramid
}

\author{
Um modelo simples para a distribuição das tensões normais da Grande Pirâmide \\ A C Tort*1 \\ ${ }^{1}$ Instituto de Física, Universidade Federal do Rio de Janeiro, Rio de Janeiro, RJ, Brasil.
}

Recebido em 8 de abril de 2016. Revisado em 10 de maio de 2016. Aceito em 15 de maio de 2016

A model for the normal stress distribution of the Great Pyramid is presented. The model is sufficiently simple as to be discussed at a basic university physics level

Keywords: statics; mechanical equilibrium.

Um modelo para a distribuição das tensões normais da Grande Pirâmide de Gizé é apresentado. O modelo é simples e pode ser discutido no ensino de física geral nos cursos básicos universtários.

Palavras-chave: estática; equilíbrio mecânico.

The Great Pyramid of Gizeh, also known as Khufu's Pyramid or Cheops' Pyramid was built between 2560 b.C.E. and 2040 b.C.E. as a tomb for pharaoh Khufu of the IV dinasty. The original dimensions were a height of 146.7 meters and a square base whose side was 230.4 meters $[1,2]$. The two mortuary rooms, the gallery, and the ascending and descending passages correspond to small fraction of the total volume and this allow us to consider the Great Pyramid as an enormous block of solid rock with a mean density approximately equal to $2500 \mathrm{~kg} / \mathrm{m}^{3}$. Several details concerning its construction can be found in [3] and references therein. The Great Pyramid give us the chance of introducing a simple quantitative model for the behavior of its normal stresses that we can discuss with our students at a moderate level of difficulty as we shall see in what comes next.

For simplicity let us suppose that this normal stress field that we shall denote by $\sigma(x, y, z)$ and measure it in $\mathrm{N} / \mathrm{m}^{2}$, is a function of $z$ only, that is $\sigma(x, y, z) \rightarrow \sigma(z)$. At the base, the force associated with this stress field must balance the total weight of the pyramid and at the vertex it must be null.

*Endereço de correspondência: tort@if.ufrj.br
Suppose that the pyramid is divided into infinitesimal truncated pyramids. Consider an element whose mass is $d m=\rho A(z) d z$, where $\rho$ is the mean density and $A(z)$ is the the area of the cross section at $z$. In equilibrium, see Figure 1

$-\sigma(z+d z) A(z+d z)+\sigma(z) A(z)-\rho g A(z) d z=0$.

Writing $\sigma(z+d z) \approx \sigma(z)+(d \sigma / d z) d z$, and $A(z+$ $d z) \approx A(z)+(d A / d z) d z$, and substituting into equation (1) we obtain up to first order

$$
\frac{d[\sigma(z) A(z)]}{d z}=-\rho g A(z)
$$

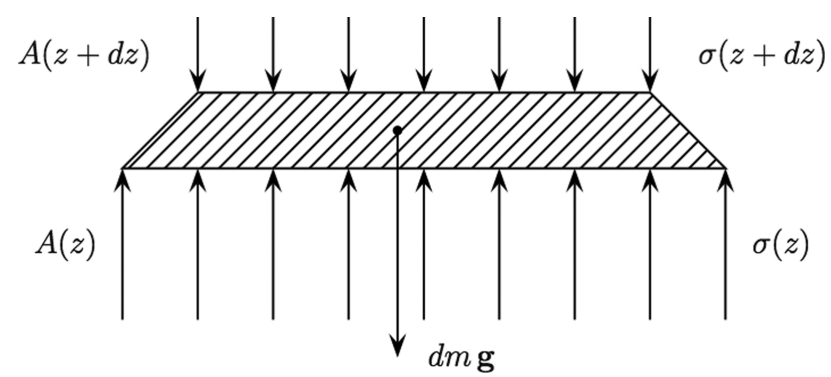

Figure 1: Infinitesimal truncated pyramid in mechanical equilibrium. 
The formal solution of this differential equation for uniform $\rho$ and $g$ is

$$
\sigma(z) A(z)=-\rho g \int A(z) d z+C,
$$

where $C$ is an integration constant. Making use of a theorem from the measure theory of volumes of cones and pyramids [4] we write

$$
\frac{A(z)}{A_{0}}=\left(\frac{H-z}{H}\right)^{2} \text {. }
$$

Taking equation (4) into equation (3) we have

$$
\sigma(z)\left(1-\frac{z}{H}\right)^{2}=-\rho g \int\left(1-\frac{z}{H}\right)^{2} d z+C
$$

Defining

$$
\omega(z)=1-\frac{z}{H}, \quad \rightarrow d z=-H d \omega,
$$

and integrating we obtain

$$
\sigma(z) \omega^{2}(z)=\rho g H \frac{\omega^{3}(z)}{3}+C .
$$

The upwards force that the ground exerts on the pyramid must balance the weight of the latter, this means that for $z=0$ we must have

$$
\sigma(0)=\frac{M g}{A_{0}}=\frac{1}{3} \rho g H .
$$

Since for $z=0, \omega(0)=1$, from equation (7) it follows that $C=0$, and the normal stress as a function of $z$ reads

$$
\sigma(z)=\frac{1}{3} \rho g H\left(1-\frac{z}{H}\right), \quad 0 \leq z \leq H .
$$

Notice that for $z=H, \sigma(H)$ is null as it should. The strength of the vertical force at the base level $\sigma(0) \times A_{0}$ is equal to $6.4 \times 10^{10} \mathrm{~N}$, that is, approximately 6400000 tonnes! This force balances the total weight of the Great Pyramid. In Figure 2. we show the graphs of the rescaled normal stress and of the rescaled cross section as functions of $z$. Notice that $\sigma(z)$ is proportional to $(1-z / H)$, and $A(z)$, is proportional to $(1-z / H)^{2}$, but the the upwards force $F(z)=\sigma(z) A(z)$ is proportional to $(1-z / H)^{3}$.

With the equations that we have at our disposal we can easily verify that a good portion of the total

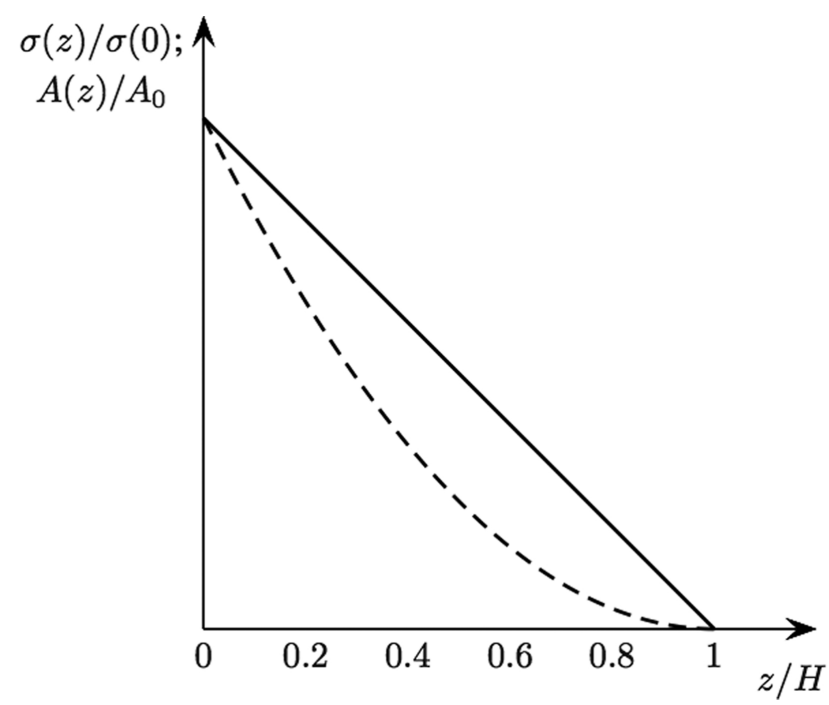

Figure 2: Plots of $\sigma(z) / \sigma(0)=(1-z / H)$, solid curve, and $A(z) / A_{0}=(1-z / H)^{2}$, dashed curve.

weight of a pyramid is kept in mechanical equilibrium by the truncated pyramid whose base is $A_{0}$ and whose height can be much less than its maximum height $H$.

The weight of the pyramid as a function of its height is given by

$$
\begin{aligned}
& P(z)=\int d m g=\rho g \int A(z) d z \\
& =\rho g A_{0} \int_{0}^{z}\left(1-\frac{z^{\prime}}{H}\right)^{2} d z^{\prime}
\end{aligned}
$$

Setting $\omega=1-z^{\prime} / H$ as before we have

$$
P(z)=\rho g H A_{0} \int_{1-\frac{z}{H}}^{1} \omega^{2} d \omega,
$$

Evaluating the integral we obtain

$$
\begin{aligned}
P(z)= & \frac{1}{3} \rho g H A_{0}\left[1-\left(1-\frac{z}{H}\right)^{3}\right] \\
= & M g\left[1-\left(1-\frac{z}{H}\right)^{3}\right], \\
& 0 \leq z \leq H .
\end{aligned}
$$

For a given $z$, the weight of the portion of the pyramid above $z$ is

$$
\begin{aligned}
& P^{*}(z)=M g-P(z)=M g\left(1-\frac{z}{H}\right)^{3}, \\
& 0 \leq z \leq H .
\end{aligned}
$$


The weight of the portion of the pyramid above $z$ must be balanced by the vertical upward force $F(z)$ associated with the normal stress at $z, F(z)=$ $\sigma(z) A(z)$. Making use of equation (9) we can easily verify that $F(z)=P^{*}(z)$.

Suppose that $z=(1 / 4) H$, that is, equal to height of the c. of $\mathrm{m}$. of the pyramid. In this case, from equation (9) we see that the normal stress is

$$
\sigma(H / 4)=\frac{1}{4} \rho g H
$$

The cross section at $z=H / 4$ is equal to $(9 / 16) A_{0}$, hence the strength of the balancing force acting on the upper part of the pyramid $(H / 4<z \leq H)$ is

$$
F(H / 4)=\frac{27}{64} M g \approx 0.42 M g
$$

that is, equal to almost half of the total weight of the Great Pyramid. This force can be considered as applied to the center of mass of upper part which lies at the height $(1 / 4) \times(3 / 4) H+(1 / 4) H=(7 / 16) H$ from the bottom, see Figure 3. Also, at $z=H / 4$, the mass of the upper part is equal to $(27 / 64)$ of the total mass of the pyramid, $M$, and that of the lower part equal to $(37 / 64) M$, as can be easily verified with the help of the equations for $P^{*}(z)$ and $P(z)$. The choice of the pyramidal shape, (or conical) means that most of the weight will be counterbalanced by the inferior layers of the structure, see the graph shown in Figure 4

The approach employed here can be easily extended to other pyramidal or conical shapes or even truncated pyramids as those that can be found in Central America.

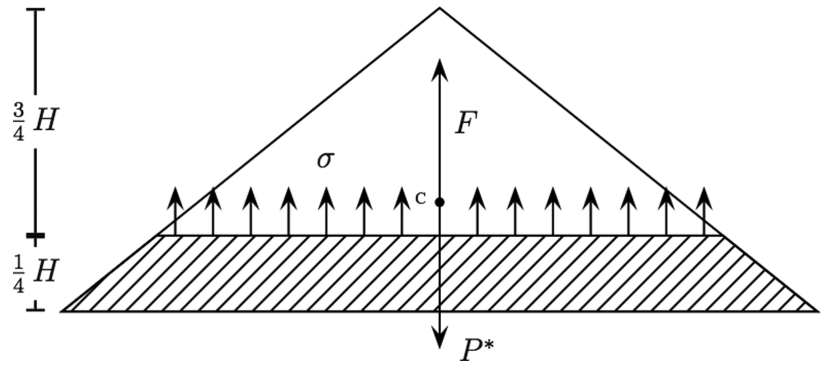

Figure 3: The vertical upward force $F(z)$ associated to the tensile stress field $\sigma(z)$ and the weight of the upper part of the pyramid, that is, $P^{*}(z)=M g-P(z)$ are shown here for $z=H / 4$. The point $C$ is the center of mass of the upper portion of the pyramid.

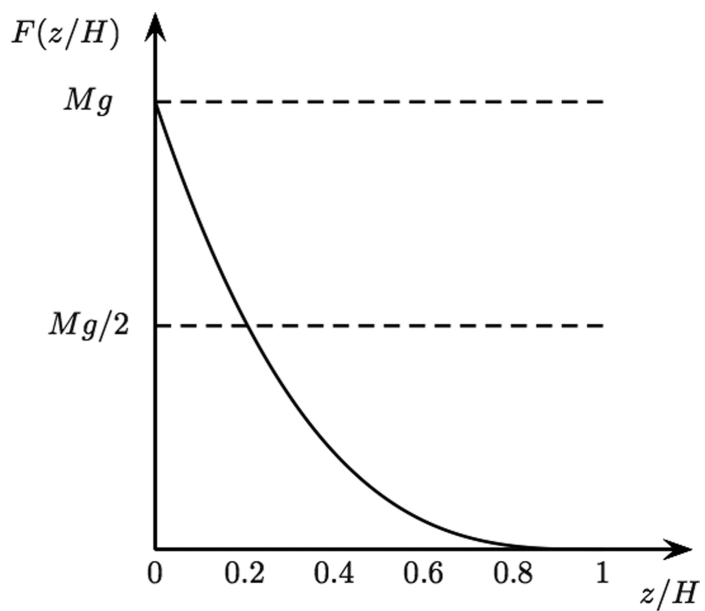

Figure 4: Force $F(z / H)=M g(1-z / H)^{3}$, exerted by the inferior portion of the pyramid on the superior one.

\section{References}

[1] M. Lehner, The Complete Pyramids of Egypt (Thames and Hudson, New York, 1997).

[2] M. Seidel e R. Schulz, Egito - Coleção Arte e Arquitetura (Dinalivro, Lisboa, 2006).

[3] A.C. Tort, Physics Education 50, 516 (2015).

[4] E. Moise, Elementary Geometry from an Advanced Standpoint (Addison-Wesley, Reading, 1963). 